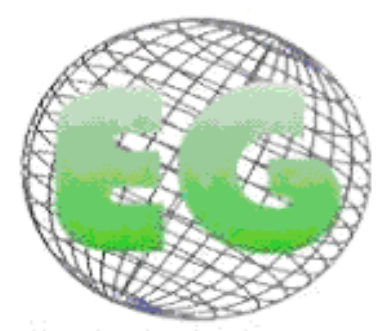

ISSN 1695-6141

N24
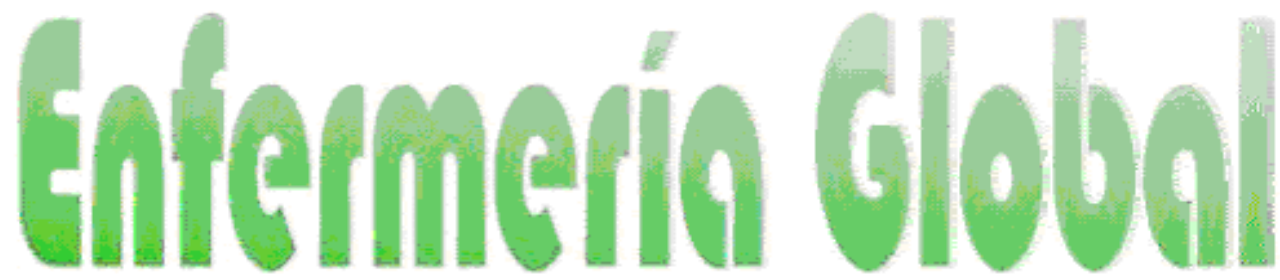

Revista electrónica trimestral de Enfermería

Octubre 2011

www.um.es/egloball

\title{
Factores asociados al incremento en el uso de la metodología enfermera en Atención Primaria
}

Factors associated to the increase in the use of nursing methodology in primary care

\author{
*Brito Brito, PR., ${ }^{* *}$ De Armas Felipe, JM., ${ }^{* * *}$ Crespo-Gómez, A., ${ }^{* * * * A g u i r r e ~}$ \\ Jaime, $A$.
}

*Enfermero consultor. Programa de Formación Continuada en Metodología enfermera. ${ }^{* *}$ Director de Enfermería. ${ }^{* * *}$ Subdirector de Enfermería. ${ }^{* * * *}$ Asesor en Metodología de la investigación. Gerencia de Atención Primaria. Tenerife.

(Este estudio fue presentado como comunicación tipo póster en el Congreso Internacional AENTDE NANDA celebrado en Madrid en mayo del año 2010).

Palabras clave: Metodología enfermera; Lenguajes estandarizados de cuidados; Historia de salud electrónica Keywords: Nursing methodology; standardised care languages; history of electronic health

\section{RESUMEN}

Introducción: Se observa un incremento sostenido en los indicadores de empleo de la Metodología enfermera (ME) en el Área de Salud de Tenerife.

Objetivo: Identificar los factores que pudieran asociarse a este crecimiento.

Material y métodos: Estudio analítico retrospectivo. Ámbito de estudio: Pacientes con historia de salud informatizada. Los sujetos del estudio fueron 609688. El análisis de los datos se llevó a cabo en el periodo temporal marzo de 2005 a febrero de 2010. Mediciones: Se analizaron, para cada Zona Básica de Salud (ZBS) y mensualmente, la frecuencia relativa de historias con Patrones funcionales de salud (PFS), diagnósticos NANDA, objetivos NOC e intervenciones NIC. Se construyó un índice global de ME con el valor medio de estos indicadores y se calculó el crecimiento a lo largo de los 60 meses a estudio. Se midieron también otras variables: utilizadores de consultas de enfermería; volumen del cupo; presión asistencial; consultas fuera de agenda prevista; consultas de otras zonas; visitas a domicilio; presencia de enfermería de enlace (ECE) en la ZBS; formación-asesoramiento en ME en la ZBS; tiempo de informatización. Procesamiento de datos: Las variables se resumieron como mediana (P5-P95) debido a su distribución no normal. La correlación entre índice-crecimiento ME y cada variable se estimó utilizando el coeficiente de correlación lineal no paramétrica de Spearman. Las comparaciones se realizaron con la prueba $U$ de Mann-Whitney. Todas las pruebas a un $5 \%$ de significación estadística.

Resultados: Se comprobó un mayor porcentaje de historias de salud con ME así como un mayor crecimiento mensual: a mayor tiempo de informatización en la zona; a mayor porcentaje de utilizadores de consultas de 
enfermería; a mayor número de visitas a domicilio; a mayor presión asistencial; a menor volumen de cupo; a menor porcentaje de consultas de otras zonas. Igualmente se comprobó mayor porcentaje de historias de salud con ME y mayor crecimiento mensual en las ZBS formadas con un programa de asesoramiento en ME así como en las zonas con ECE.

Conclusiones: El incremento en el empleo de la ME en las ZBS de Tenerife se asocia a cada uno de los factores considerados, en diferente sentido y magnitud.

\section{ABSTRACT}

Introduction: Indicators show a sustained increase in the use of nursing methodology (NM) in the Health Complex in Tenerife.

Aim: to identify the factors that may be associated with this growth.

Methods and materials: Retrospective analytical study. Area of study: patients with a computerised record of health. Subjects of the study numbered 609,688. Data analysis was performed from March 2005 to February 2010. Measurements: these were analysed monthly for each Basic Health Area (BHA) and measured the relative frequency of records with functional health patterns, (PFS), NANDA diagnoses, NOC objectives and NIC interventions. An NM global index was constructed from the mean value of the indicators and growth was calculated over the 60 months studied. Other variables measured included users of nursing consultations; quotas; patient load; non programmed consultations; consultations from other areas; home visits; presence of link nursing in the BHA; training and assessment in ME in the BHA; computer time. Data processing: variables were summarised as median (P5-P95) due to the non normal distribution. The correlation índex-ME growth and each variable was estimated using the Spearman non parametric linear correlation coefficient. Comparisons were made with the Mann-Whitney U test. All tests were significant at $5 \%$.

Results: Findings show a greater percentage of health records with ME and greater monthly growth when computerisation in the area was longer; when the percentage of users of nursing consultancies was higher; when the number of home visits was higher; when patient load was greater; when quotas were smaller; when consultations from other areas were lower. There was a higher percentage of medical records with ME and greater monthly growth in BHAs with ME assessment programme and in areas with ECE.

Conclusions: Increased use of $\mathrm{ME}$ in the BHA in Tenerife is associated to each of the factors considered in different direction and size.

\section{INTRODUCCIÓN}

El incremento en la práctica asistencial del uso de lenguajes estandarizados de cuidados NANDA, NOC y NIC (NNN) ha sido constante en los últimos años. En el ámbito de la Atención Primaria (AP) española se han puesto en marcha diversas iniciativas para integrar el uso de la Metodología enfermera (ME) en la práctica clínica ${ }^{(1)(2)(3)(4)(5)}$. Además, todo ello ha recibido el impulso de la informatización de las historias de salud, las cuales cuentan con módulos específicos de ME y lenguajes NNN.

Las dificultades para la práctica clínica con lenguajes estandarizados de cuidados están descritas por diversos autores ${ }^{(6)(7)(8)}$ tanto a nivel nacional como internacional y tienen que ver sobre todo con la imprecisión de las etiquetas diagnósticas, la traducción y adaptación cultural de los problemas que describen, la falta de práctica, la presión asistencial y las cargas de trabajo. A todo lo anterior se suma la necesidad del conocimiento y manejo de una nueva herramienta: la historia de salud informatizada.

Los registros de salud electrónicos han resultado beneficiosos para introducir en la práctica estos lenguajes de cuidados ${ }^{(9)(10)}$ siendo muy útiles para el desarrollo de los mismos y permitiendo incluso, como mostró Pérez Rivas et al, realizar explotaciones de datos orientadas a la consecución de objetivos en salud mediante el uso de dichos lenguajes.

La implantación del programa de historia informatizada Drago AP en el Área de Salud de Tenerife comenzó en el año 2004 y finalizó en 2008. En esta Área trabajan 574 enfermeras distribuidas en 39 Zonas Básicas de Salud (ZBS). 
El módulo de ME se incluyó en la historia informatizada en marzo de 2005 con una estructura de valoración por Patrones Funcionales de Salud de Gordon (PFS), la clasificación diagnóstica NANDA, la de resultados NOC y la de intervenciones NIC. En los meses y años sucesivos, este módulo ha sido modificado prestando nuevas opciones para el trabajo en consulta. Actualmente es posible escribir comentarios a las etiquetas y al plan de cuidados, añadir las características definitorias y los factores relacionados de las etiquetas diagnósticas, los indicadores de las etiquetas de resultados y las actividades de las etiquetas de intervención.

La utilización del módulo de ME en el Área de Salud de Tenerife no fue muy alta en los primeros años de implantación ${ }^{(9)}$, incrementándose considerablemente sobre todo en 2008 y $2009^{(10)}$

Se ha observado un incremento sostenido en los indicadores de empleo de la Metodología Enfermera (ME) en el Área de salud de Tenerife.

El objetivo de este trabajo es identificar los factores que pudieran asociarse a este crecimiento.

\section{MATERIAL Y MÉTODOS}

Estudio analítico retrospectivo.

Ámbito de estudio: Pacientes con historia de salud informatizada atendidos en Atención Primaria en el Área de Salud de Tenerife.

Los sujetos del estudio fueron 609688: número de historias informatizadas a 1 de marzo de 2010.

El análisis de los datos se llevó a cabo sobre un total de 60 meses comprendidos en el periodo de marzo de 2005 a febrero de 2010 (ambos inclusive).

Mediciones: Se calculó, para cada ZBS y para todo el Área, la frecuencia relativa mensual de historias con PFS, diagnósticos NANDA (Dde), objetivos NOC e intervenciones NIC. Se construyó un índice de uso global de la ME con el valor medio mensual de estos indicadores. Se calculó también el crecimiento mensual de todas las variables anteriores. Además se consideraron mensualmente, para cada ZBS y para todo el Área las siguientes variables: frecuencia relativa de utilizadores de consultas de enfermería; volumen del cupo; presión asistencial; frecuencia de consultas fuera de agenda prevista; frecuencia de consultas de otras ZBS; frecuencia de visitas a domicilio; presencia de enfermería de enlace en la zona (ECE); formación en ME en la zona; tiempo de informatización.

Procesamiento de datos: Las variables se resumieron como mediana (P5-P95) debido a su distribución no normal. La correlación entre índice-crecimiento ME y las variables anteriores se estimó utilizando el coeficiente de correlación lineal no paramétrica de Spearman. Las comparaciones se realizaron con la prueba $U$ de Mann-Whitney. Todas las pruebas se realizaron a un $5 \%$ de significación estadística.

Los datos se obtuvieron mediante explotación del programa de historia de salud informatizada Drago AP previo permiso solicitado a la Dirección de Enfermería de la Gerencia de Atención Primaria de Tenerife. Se construyó una base de datos excel con las variables a estudio para el volcado. 
El análisis estadístico se realizó mediante el aplicativo SPSS para Windows.

\section{RESULTADOS}

Concluido el mes de febrero de 2010, en el Área de Salud de Tenerife había un total de 95653 historias con PFS (14\% sobre el total de historias informatizadas); 298687 historias con Dde (43\%); 219936 historias con objetivos NOC (31,5\%) y 214814 historias con intervenciones NIC (31\%). Al observar el valor de la variable construida como índice de uso global de la ME, esta se sitúa en un $30 \%$. En la misma fecha, el porcentaje de utilizadores de consultas de enfermería en los últimos doce meses fue de un $48 \%$ sobre el total de tarjetas sanitarias individuales. En ningún momento del periodo estudiado esta cifra de frecuentadores fue más elevada.

El porcentaje en el Área de historias informatizadas con PFS fue de un $0,2 \%$ en febrero de 2006; $1,3 \%$ en febrero de 2007; 3,8\% en febrero de 2008 y $7 \%$ en febrero de 2009 . El porcentaje de historias con Dde fue de un $0,3 \%$ en el mismo mes de 2006; $1,9 \%$ en 2007 ; $9,7 \%$ en 2008 y $25,7 \%$ en 2009. Si centramos los datos de ME observando el comportamiento del índice de uso global de la ME en los periodos anuales señalados anteriormente comprobamos un $0,2 \% ; 1,6 \% ; 6,5 \%$ y $17 \%$.

La evolución mensual en el período estudiado de cada variable puede observarse en los Gráficos 1 al 6.

\section{Gráfico 1}

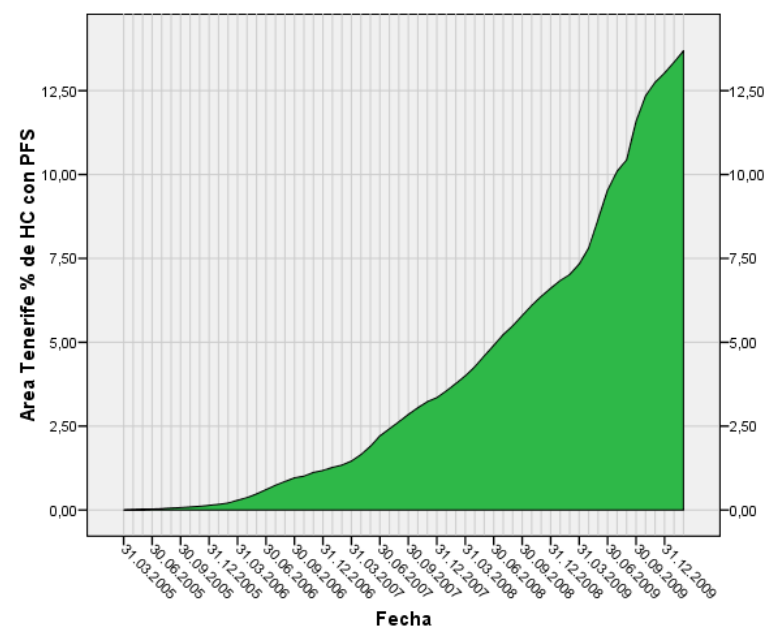




\section{Gráfico 2}

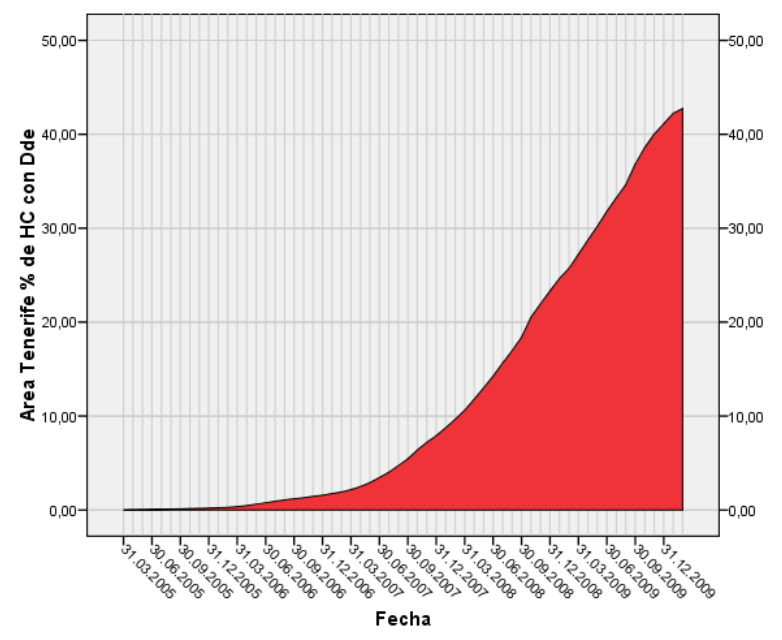

\section{Gráfico 3}

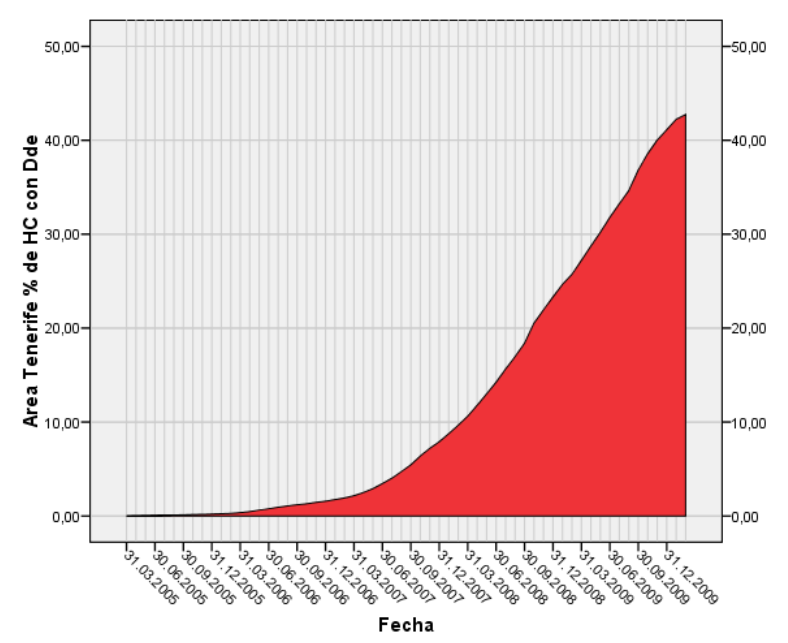




\section{Gráfico 4}

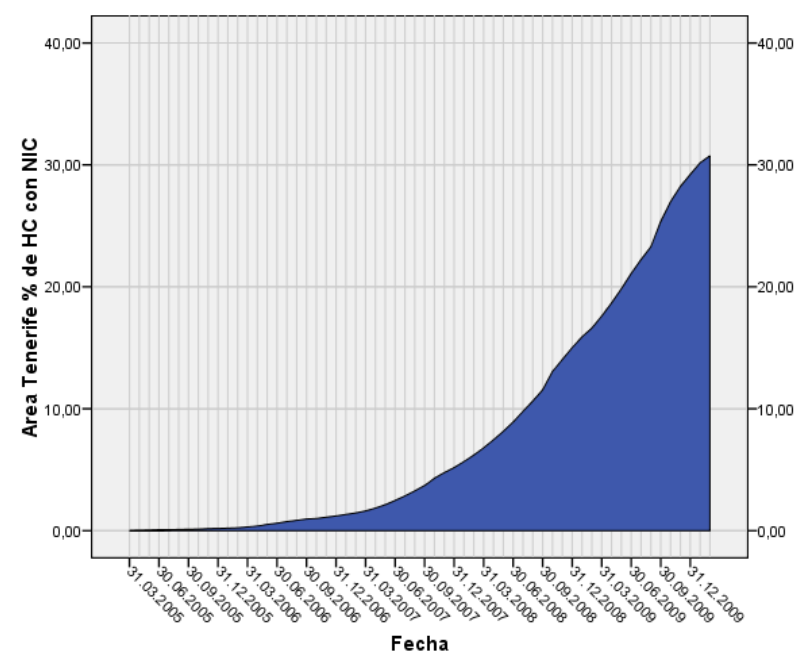

Gráfico 5

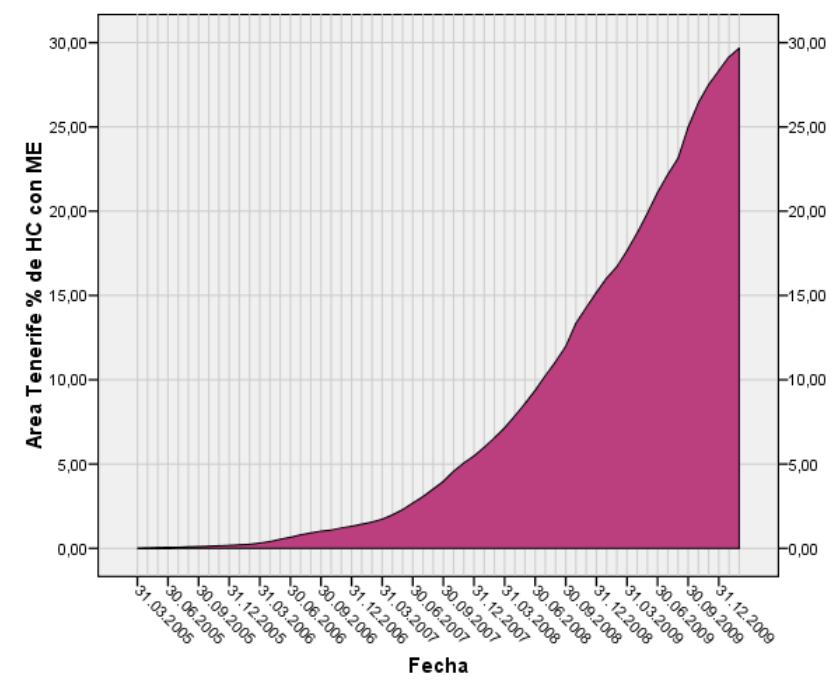




\section{Gráfico 6}

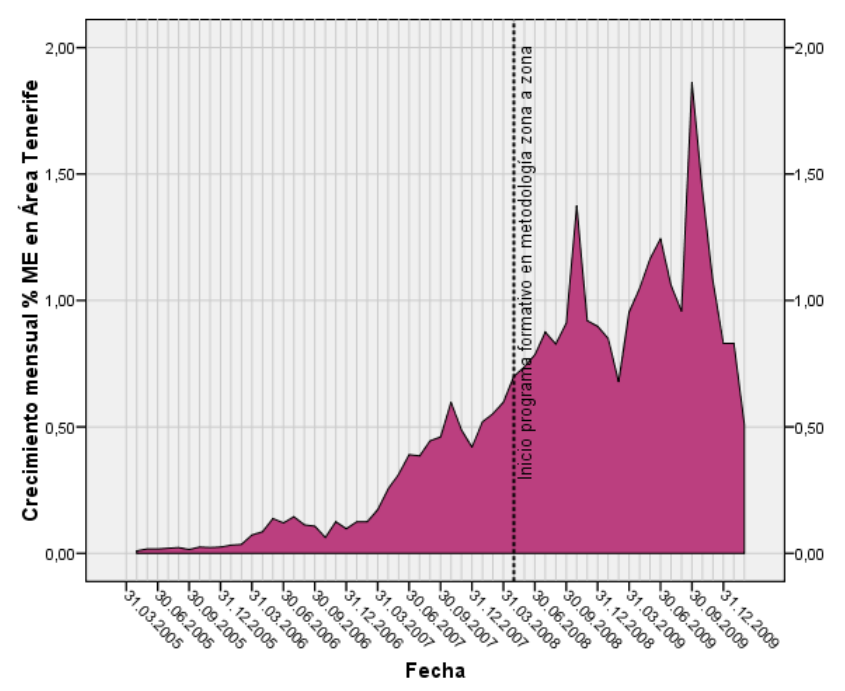

La cifra de uso de PFS se vio prácticamente duplicada cada año desde 2007. En cuanto a los Dde, su utilización se vio casi triplicada entre 2008 y 2009 y duplicada entre 2009 y 2010.

La variable índice de uso global de la ME experimentó el mismo crecimiento que los Dde.

Se comprobó un mayor porcentaje de historias de salud con lenguajes estandarizados de cuidados así como un mayor crecimiento mensual:

- A mayor tiempo de informatización en la zona.

- A mayor porcentaje de utilizadores de consultas de enfermería.

- A mayor número de visitas a domicilio.

- A mayor presión asistencial.

- A menor volumen de cupo.

- A menor porcentaje de consultas de otras zonas de salud. 
Tabla 1. Factores relacionados con \% de HC con ME

\begin{tabular}{|lccc|}
\hline \multicolumn{1}{|c}{ Factores } & $\mathrm{n}$ & rho & $\mathrm{p}$ \\
\hline Tiempo de informatización & 2340 & 0,893 & $<0,001$ \\
\hline Utilizadores de enfermería & 2308 & 0,712 & $<0,001$ \\
\hline Visitas a domicilio & 2167 & 0,418 & $<0,001$ \\
\hline Presión asistencial & 2167 & 0,338 & $<0,001$ \\
\hline $\begin{array}{l}\text { Consultas de otras zonas de } \\
\text { salud }\end{array}$ & 2340 & $-0,379$ & $<0,001$ \\
\hline
\end{tabular}

Tabla 2. Factores relacionados con crecimiento mensual \% de $\mathrm{HC}$ con ME.

\begin{tabular}{|lccc|}
\hline \multicolumn{1}{|c}{ Factores } & $\mathrm{n}$ & rho & $\mathrm{p}$ \\
\hline Tiempo de informatización & 2301 & 0,725 & $<0,001$ \\
\hline Utilizadores de enfermería & 2269 & 0,592 & $<0,001$ \\
Visitas a domicilio & 2141 & 0,351 & $<0,001$ \\
\hline Presión asistencial & 2141 & 0,313 & $<0,001$ \\
\hline Volumen de cupo & 2301 & $-0,366$ & $<0,001$ \\
\hline $\begin{array}{l}\text { Consultas de otras zonas de } \\
\text { salud }\end{array}$ & 2141 & $-0,63$ & $<0,005$ \\
\hline
\end{tabular}

Igualmente, se comprobó mayor porcentaje de historias de salud con ME y mayor crecimiento mensual en las zonas de salud formadas con un programa de asesoramientoentrenamiento específico para el uso de lenguajes PFS-NNN así como en las zonas con enfermería comunitaria de enlace. El crecimiento mensual en las zonas con ECE fue dos veces mayor que en las zonas sin ECE (Tabla 3). El crecimiento mensual en las zonas formadas en ME fue cuatro veces mayor que en las zonas no formadas (Tabla 4). 
Tabla 3. Relaciones entre variables ECE y \% de HC con ME-crecimiento mensual

\begin{tabular}{|ccc|}
\hline Factores & Mediana (P5-P95) & $p$ \\
\hline HC con ME sin enfermería de enlace & $2,25(0-28) \%$ & $<0,001$ \\
HC con ME con enfermería de enlace & $7,25(0-33) \%$ & $<0,001$ \\
\hline Crecimiento mes ME sin enfermería de & $0,25(0-1,74) \%$ & $<0,001$ \\
enlace & & \\
\hline $\begin{array}{c}\text { Crecimiento mes ME con enfermería de } \\
\text { enlace }\end{array}$ & $0,5(0-1,5) \%$ & $<0,001$ \\
\hline
\end{tabular}

Tabla 4. Relaciones entre variables intervención formativa y \% de HC con MEcrecimiento mensual

\begin{tabular}{|lcc|}
\hline \multicolumn{1}{|c}{ Factores } & Mediana (P5-P95) & $\mathrm{p}$ \\
\hline HC con ME pre-intervención formativa & $2,5(0-30) \%$ & $<0,001$ \\
\hline HC con ME post-intervención formativa & $20,7(10,5-31) \%$ & $<0,001$ \\
\hline $\begin{array}{l}\text { Crecimiento mes ME pre-intervención } \\
\text { formativa }\end{array}$ & $0,25(0-1,5) \%$ & $<0,001$ \\
\hline $\begin{array}{l}\text { Crecimiento mes ME post-intervención } \\
\text { formativa }\end{array}$ & $1(0,36-2) \%$ & $<0,001$ \\
\hline
\end{tabular}

\section{DISCUSIÓN Y CONCLUSIONES}

Este estudio muestra, a la luz de los resultados obtenidos, cómo diferentes factores relacionados con la práctica asistencial influyen de manera significativa, y en diferentes sentidos y magnitudes, en la utilización y registro de los lenguajes de cuidados estandarizados.

Dichos factores pueden ser tenidos en cuenta a la hora de implementar un programa formativo de Metodología enfermera.

Es natural entender que el proceso de aprendizaje de nuevas herramientas de trabajo en consulta (lenguajes NNN e historia informatizada) conlleva un tiempo prudencial que puede durar meses e incluso años. Parece conveniente, antes de realizar explotaciones clínicas de datos en el Área estudiada, esperar a que transcurra algo más de tiempo tras la implantación de dichas herramientas.

Como limitaciones del presente estudio, debemos tener en cuenta que los datos explotados son puramente cuantitativos, siendo conveniente realizar futuras investigaciones que 
complementen nuestras conclusiones y que se basen en datos cualitativos. Además, no fue posible, dadas las características de la explotación, comprobar el número de historias con los cuatro parámetros al mismo tiempo PFS, Dde, NOC, NIC. Por ello, se creó el índice de uso global de la ME.

Es relevante destacar cómo, considerando el porcentaje de frecuentadores en consultas de enfermería, este índice se incrementa de manera significativamente considerable. Así, podemos concluir que, siendo aproximadamente la mitad de los cupos el porcentaje de personas que visita a lo largo del año la consulta de su enfermera, y observando el índice de uso global de la ME en febrero de 2010 en un 30\%, la cifra real de utilización de lenguajes estandarizados de cuidados se vería duplicada.

Interesa realizar una implementación cuidadosa de los lenguajes de cuidado en la práctica asistencial. Müller Staub ${ }^{(13)}$ apunta que ello conlleva la elección de diagnósticos más precisos, intervenciones más efectivas y una mejora en la consecución de los resultados. Se hacen necesarias también investigaciones orientadas a mejorar la precisión del diagnóstico enfermero que mejoren la concordancia entre profesionales a la hora de determinar la presencia de los mismos ${ }^{(14)(15) .}$

\section{BIBLIOGRAFÍA}

(1) Pérez Rivas FJ, Ochandorena MS, Santamaría JM, García M, Solano V, Beamud M, Gil de Pareja MJ. Aplicación de la metodología enfermera en atención primaria. Rev Calidad Asistencial. 2006;21(5):247-54.

(2) Izquierdo Carrasco JM, Pérez Badía MB, Ramírez Rodríguez FJ, Serrano Escalona I, Torres Montero MD, Conde Lara G. Implantación del proceso enfermero. Rev Rol Enferm. 2002; 25(7-8):8-13.

(3) Raña Lama, CD. Diagnósticos de Enfermería: Líneas de Investigación en Atención Primaria de Salud. Enferm Cient. 1999;208-9:28-30.

(4) Novo MM, Rodríguez JA, Sicilia I, Izquierdo D, Daroqui P. Proceso de implantación de Diagnósticos de Enfermería en EAP de la provincia de Santa Cruz de Tenerife. Index Enferm. 1995;IV:92.

(5) Martín E, García F, Asenjo A. Diagnósticos de Enfermería. Estrategias de implantación. Rev Rol Enferm. 1997;225:57-62.

(6) Montesinos Afonso, NM; Duarte Clíments, G; Sánchez Gómez, MB. Diagnósticos enfermeros. Proceso y taxonomía diagnóstica. En: Duarte Clíments, G; Gómez Salgado, J; Sánchez Gómez, MB. Fundamentos teóricos de la enfermería comunitaria. Madrid: Enfo, 2007:293-311.

(7) Paganin A, Moraes MA, Pokorski S, Rabelo ER. Factors that inhibit the use of nursing language. Int J Nurs Terminol Classif. 2008; 19(4):150-157.

(8) Thomas NM, Newsome GG. Factors affecting the use of nursing diagnosis. Nurs Outlook. 1992; 40(4):182-186.

(9) Lunney, M; Delaney, C; Duffy, M; Moorhead, S; Welton, J. Advocating for standardized nursing languages in electronic health records. Journal of nursing administration. 2005; 35:1 3.

(10) Westra, BL; Delaney, CW; Konicek, D; Keenan, G. Nursing standards to support the electronic health record. Nursing outlook. 2008; 56(5):258-266.

(11) Barrios Torres R, Estupiñán Ramírez M, De la Huerga Moreno S, López Cavero J, Navarro Brito E. Patrones funcionales y diagnósticos de enfermería más frecuentes en DRAGO-AP. Actas del $6^{\text {th }}$ Congreso de la Asociación de Enfermería Comunitaria (AEC) Simposium Internacional de Enfermería Comunitaria; 2006 Nov; Valencia, España. 
(12) Brito Brito PR, Aguirre-Jaime A, de Armas Felipe JM, Crespo Gómez A. Tendencia en el uso de la metodología enfermera en un Área de Salud. Póster. Congreso Internacional AENTDE-NANDA 2010. Hacia un futuro global para enfermería: desarrollar el lenguaje compartiendo conocimientos; 2010 May; Madrid, España.

(13) Müller-Staub M. Studies about use and application of nursing classifications. Pflege Z. 2009; 62(6): 354-9.

(14) Lunney, M. Valoración, juicio clínico y diagnósticos enfermeros: cómo determinar los diagnósticos adecuados. En: Nanda-International. Diagnósticos enfermeros. Definiciones y clasificación 2009-2011. Barcelona: Elsevier, 2010:3-17.

(15) Lunney, M. Critical need to address accuracy of nurses'diagnoses. The online journal of issues in nursing. 2008; 13(1). Disponible en: $<$ http://www.nursingworld.org/MainMenuCategories/ANAMarketplace/ANAPeriodicals/OJIN/T ableofContents/vol132008/No1Jan08/ArticlePreviousTopic/AccuracyofNursesDiagnoses.asp $\underline{x}>$ [Consultado el 03.04.2010] 